\title{
Incidence Tendency Analysis on Type 2 Diabetes in 4 Asian Countries - China, Malaysia, Singapore, and Thailand, 1990-2019
}

\author{
Wenhui Shi'; Qiqi Wang²; Jiaojiao Zhang ${ }^{3}$; Jingwen Zhang'; Feng Tan ${ }^{5, *}$; Shicheng $\mathrm{Yu}^{2, *}$
}

\section{Summary}

What is already known about this topic?

Roughly $80 \%$ of the global disease burden caused by diabetes comes from low-and-middle income countries, and $60 \%$ of diabetics are located in Asia; 6 of the top 10 countries with the highest prevalence of diabetes are in Asia.

\section{What is added by this report?}

Compared with 1990, the growth rate of the standardized incidence rate of type 2 diabetes in 2019 in China was significantly lower than that of the entire world, while Malaysia, Singapore, and Thailand all had a negative growth rate in incidence. A great difference was found by the 5-year growth of the standardized incidence rate of type 2 diabetes from 1990 to 2019 in the 4 selected countries.

What are the implications for public health practices?

Compared with the measures taken for comprehensive prevention and control of risk factors in Singapore, there is still a lot of work that needs to be done in China, Malaysia, and Thailand.

The International Diabetes Federation (IDF) has pointed out that $60 \%$ of diabetics are from Asia (1). Moreover, 6 of the top 10 countries with the highest prevalence of diabetes are also in Asia. The 30 years results of Daqing's diabetes prevention and control program in China showed that a healthy lifestyle has a long-term effect on diabetes prevention (2). In this article, 4 Asian countries were selected (China, Malaysia, Singapore, and Thailand) that have taken measures to prevent and control diabetes at the national level and also have the largest ethnically Chinese populations in Asia.

All data used in this article, i.e., the incidence of type 2 diabetes, were fetched from Global Burden of Disease (GBD) 2019 (3-4), and the standardized incidence rates (IRs, /100,000) was calculated based on the World Standard Population in 2019 provided by GBD 2019 (3). The growth rate, 5-year growth, and annual speed of increase between 1990 to 2019 were adopted as a metric to represent and evaluate the tendency of the standardized incidence rate along with time. Based on the results and analyses, evidence and guidance are provided for implementing and evaluating diabetes prevention and control.

Table 1 showed that there was a huge difference in the changes of the standardized incidence, e.g., growth rate and annual speed of increase, from 1990 to 2019 between the 4 Asian countries and the entire world. The global standardized incidence rate has increased by more than $40 \%$. The increase of the incidence in China, with a growth rate of $6.26 \%$, was significantly lower than that of the world overall. Although differing in the specific values, Malaysia, Singapore, and Thailand all have had a negative growth rate in incidence; particularly, the incidence growth rates were $-50.02 \%,-86.88 \%$, and $-26.75 \%$, respectively.

As shown in Table 2, like the former observation, the 5-year growth of the standardized incidence from 1990 to 2019 differed greatly among the 4 Asian countries. Specifically, China has an overall increasing trend, and its growth rate fluctuates greatly. A negative growth period could be observed in 1990-1995 and 2005-2015, during which its overall growth rate gradually slowed down, and there was no incremental change from 2015 to 2019. The annual speed of increase of Malaysia showed a rapid decrease on average, but it rebounded from 2015 to 2019 with an annual speed of increase of $3.69 \%$. The annual speed of increase of Singapore was also continuously decreasing, where a rapid decline could be seen during 2005-2015, with a decreasing rate of $15 \%$ approximately. However, the speed of decline in Singapore was reduced to $0.82 \%$ during $2015-2019$. In general, the decreasing rate of Singapore was quite higher than that of Malaysia from 1990 to 2019. The annual speed of increase of Thailand presented an 
TABLE 1. The standardized incidence rate of type 2 diabetes and its changes worldwide, 1990-2019.

\begin{tabular}{|c|c|c|c|c|c|}
\hline \multirow{2}{*}{ Nations } & \multicolumn{2}{|c|}{ Standardized incidence rate $(/ 100,000)$} & \multicolumn{3}{|c|}{ Changes of 2019 relative to 1990} \\
\hline & 1990 & 2019 & Increment $(/ 100,000)$ & Growth rate $(\%)$ & Annual speed of increase (\%) \\
\hline Worldwide & 184.55 & 259.94 & 75.39 & 40.85 & 1.82 \\
\hline China & 8.63 & 9.17 & 0.54 & 6.26 & 0.32 \\
\hline Malaysia & 26.57 & 13.28 & -13.29 & -50.02 & -3.58 \\
\hline Singapore & 18.22 & 2.39 & -15.83 & -86.88 & -10.14 \\
\hline Thailand & 24.90 & 18.24 & -6.66 & -26.75 & -1.62 \\
\hline
\end{tabular}

TABLE 2. The 5-year growth of the standardized incidence rate in China, Singapore, Malaysia, and Thailand, 1990-2019.

\begin{tabular}{|c|c|c|c|c|c|c|c|c|c|c|c|c|}
\hline \multirow{2}{*}{ Year } & \multicolumn{3}{|c|}{ China } & \multicolumn{3}{|c|}{ Malaysia } & \multicolumn{3}{|c|}{ Singapore } & \multicolumn{3}{|c|}{ Thailand } \\
\hline & IRs & $\Delta I R s$ & $A S I$ & IRs & $\Delta I R s$ & $A S I$ & IRs & $\Delta I R s$ & $A S I$ & IRs & $\Delta I R s$ & $A S I$ \\
\hline 1990 & 8.63 & - & - & 26.57 & - & - & 18.22 & - & - & 24.90 & - & - \\
\hline 1995 & 8.43 & -0.20 & -0.47 & 25.44 & -1.13 & -0.87 & 12.82 & -5.40 & -6.79 & 26.73 & 1.83 & 1.43 \\
\hline 2000 & 9.40 & 0.97 & 2.20 & 24.85 & -0.59 & -0.47 & 12.60 & -0.22 & -0.35 & 24.58 & -2.15 & -1.66 \\
\hline 2005 & 10.65 & 1.25 & 2.53 & 20.98 & -3.87 & -3.33 & 11.59 & -1.01 & -1.66 & 18.64 & -5.94 & -5.38 \\
\hline 2010 & 9.54 & -1.11 & -2.18 & 13.77 & -7.21 & -8.08 & 5.39 & -6.20 & -14.20 & 16.59 & -2.05 & -2.30 \\
\hline 2015 & 9.17 & -0.37 & -0.79 & 11.49 & -2.28 & -3.56 & 2.47 & -2.92 & -14.45 & 17.51 & 0.92 & 1.09 \\
\hline $2019^{*}$ & 9.17 & 0.00 & 0.00 & 13.28 & 1.79 & 3.69 & 2.39 & -0.08 & -0.82 & 18.24 & 0.73 & 1.03 \\
\hline
\end{tabular}

Note: - means the growth and the annual speed of increase in 1990 cannot to be calculated.

Abbreviations: IRs=standardized incidence rate $(1 / 100,000) ; \Delta I R s=5$-year growth of the standardized incidence $(1 / 100,000) ; A S I=a n n u a l$ speed of increase over the last 5 years of the standardized incidence $(\%)$.

* The growth and the annual speed of increase in 2019 were computed based on the 4-year change with respect to 2015.

overall downward tendency but with a non-negligible fluctuation: it maintained its decline from 1995 to 2010, but it rebounded during 2010-2015, with an annual speed of increase of $1.09 \%$, and then maintained the annual speed of $1.03 \%$ from 2015 to 2019.

Figure $1 \mathrm{~A}, 1 \mathrm{~B}$, and $1 \mathrm{C}$ showed that the standardized incidence rate of different age groups in the 4 Asian countries differed. The country that had the lowest standardized incidence rate for all 3 age groups (i.e., $15-44,45-59$, and above 60 years old) in 1990 was China, but Singapore had the lowest incidence in 2019. In summary, the age group of 15-44 years had the lowest standardized incidence rate of type 2 diabetes $(<0.5 / 100,000)$, while the age group of above 60 years old had the highest one $(<20 / 100,000)$.

According to the change from 1990 to 2019, for the age group of 15-44 years, Malaysia had the largest decline in the standardized incidence rate, with a decrease of $0.61 / 100,000$, followed by Singapore, with a decrease of $0.58 / 100,000$. China only had a decrease of $0.02 / 100,000$, and Thailand even increased $0.17 / 100,000$. For the age group of 45-59 years, Malaysia and Singapore attained the largest decline in the standardized incidence rate, with a decrease of
$16.10 / 100,000$ and 16.01/100,000, respectively, followed by Thailand, with a decrease of 10.58/ 100,000. China had the smallest decrease, i.e., 2.79/100,000; For the age group of above 60 years old, Singapore made the largest decline, followed by Malaysia and Thailand, with a decrease of $99.76 / 100,000,81.11 / 100,000$, and 35.01/100,000, respectively. China had a decline that was much lower than the above three countries, only a decrease of $7.47 / 100,000$.

Therefore, this article conducted an analysis of the tendency of incidence with respect to different age groups. In terms of the age group of 45-59 years, as plotted in Figure 1B, the standardized incidence rate of type 2 diabetes in the 4 Asian countries showed overall decreases. Among the 4 countries, Singapore had an always-decreasing curve and had the most significant decline, but its downward tendency was relieved after the low point in 2013. Malaysia and Thailand both had a relatively large decline, which was always higher than that of China, but with fluctuations. The incidence for China remained relatively stable, just having a slight decline.

However, of the age group above 60 years old, as shown in Figure 1C, only Singapore showed an 

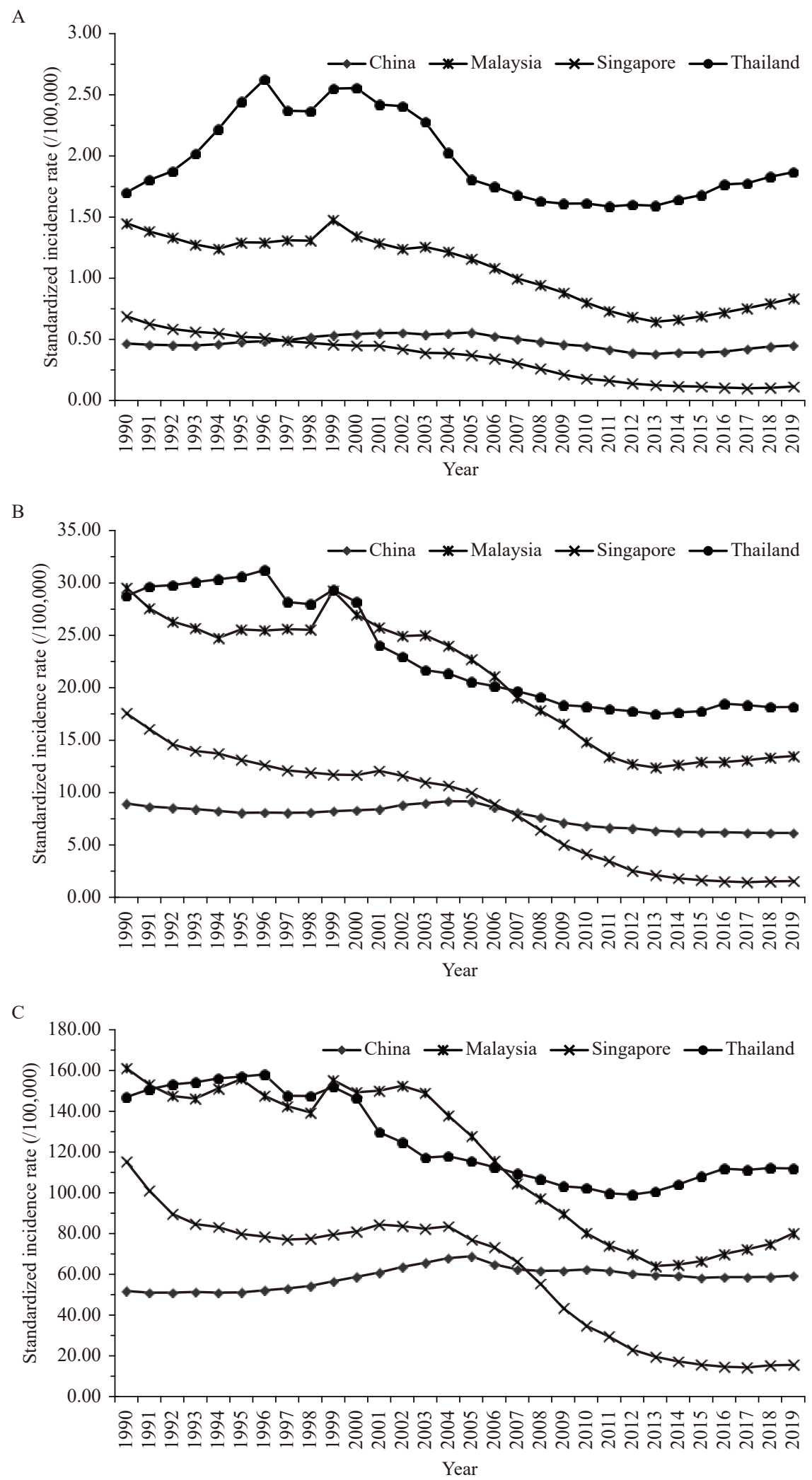

FIGURE 1. The standardized incidence rate of type 2 diabetes in different age groups in the 4 countries, 1990-2019. (A) 15-44 years; (B) 45-59 years; (C) 60 years.

Note: There are 4 countries shown in Figure 1C; however, in order to highlight the main findings, only the lowest standardized incidence rate of type 2 diabetes in the age group of 60 years old and above in 1990 and 2019 (China and Singapore, respectively) were described in the corresponding text. Malaysia and Thailand were described in other parts. 
obvious downward trend. Malaysia had a significant rebound since 2013 after a decrease with fluctuation. After an early-stage reduction, the incidence rate of Thailand started to fluctuate and increase slightly in the high incidence rate range, i.e., roughly from $110 / 100,000$ to $130 / 100,000$. The incidence rate in China was lower than in Malaysia and Thailand, but the overall trend showed a slow rise.

\section{DISCUSSIONS}

The incidence of diabetes from 1990 to 2019 in 4 Asian countries decreased according to the GBD 2019 in 4 Asian countries. The difference in the incidence of diabetes from 1990 to 2019 in the 4 Asian countries provides evidence and guidance for the implementation and evaluation of diabetes prevention and control measurements. However, the continuous decreases in the past 30 years in Singapore showed a successful example of diabetes control at the national level, which can be described as a comprehensive strategy and is worth consideration in other countries, including risk factor control, guarantee of funding, laws, National Healthy Lifestyle Project (5) in the whole society, and the consistent adoption of comprehensive measures.

Although healthy lifestyle strategies for the prevention and control of diabetes were undertaken in the 4 countries (G), the specifics were different. The measures taken in Thailand included the practice of prohibiting smoking (7) since 1953, the "Alcohol Control Law" promulgated since 2008, and advocating reducing salt and sodium intake and encouraging people to increase exercise. Malaysia in the 1970s had implemented schools' nutrition programs for students since the 1970s (8), and smoking was prohibited in public places and religious sites. In China, such measures as tobacco control, "China Healthy Lifestyle for All"(9), "National Nutrition Plan (2017-2030)," and Healthy China were continuously carried out from a national perspective.

The incidence tendency of type 2 diabetes in different groups meant different measures should be taken according to the characteristics of different groups. At present, China is the only country whose incidence rate is on the rise, and the incidence of diabetes in the 60 years and over group was much higher than that in 18-44 years group, which means that China still has the most diabetes patients of the 4 countries. China is also facing population aging, which may cause more diabetes and a higher burden, more measures should be learned from Singapore immediately. Meanwhile, although this study found that the elderly were a key group for diabetes prevention and control, young people should not be ignored, and early interventions for young people may achieve more benefits.

This study was subject to some limitations. First, this study used GBD 2019 data to ensure comparability of the four countries, but the actual circumstances may differ from the actual incidence due to limitations in GBD methodology, e.g., data source and calculation methods. If real data could be obtained from an intelligent crowd monitoring system in the future, the results and analyses derived from this article can be further verified and refined. Second, the sampling, diagnosis, and statistical analysis of diabetes have an important influence on the estimation of diabetes incidence; because of the difference in healthcare services between the elderly and adolescents, elderly patients are more likely to be diagnosed, and the incidence of diabetes in adolescents is more likely to be underestimated. More research and proactive surveillance are needed to help us learn the current circumstances and trends of the burden of type 2 diabetes in adolescents.

Funding: Key Research and Development Programme [2018YFC1315305].

doi: $10.46234 / \mathrm{ccdcw} 2021.268$

\# Corresponding authors: Feng Tan, tanfeng@chinacdc.cn; Shicheng Yu, yusc@chinacdc.cn.

1 Division of Non-communicable Diseases and Elderly Health Management, Chinese Center for Disease Control and Prevention, Beijing, China; ${ }^{2}$ Office of Epidemiology, Chinese Center for Disease Control and Prevention, Beijing, China; ${ }^{3}$ Institute of Infectious Diseases, Beijing Center for Disease Control and Prevention, Beijing, China; ${ }^{4}$ Shijiazhuang Finance Bureau of Hebei Province, Shijiazhuang, Hebei, China; 5 Division of Academic Publishing Management, Chinese Center for Disease Control and Prevention, Beijing, China.

Submitted: October 27, 2021; Accepted: December 21, 2021

\section{REFERENCES}

1. IDF. IDF diabetes atlas 10th edition. 2021. https://diabetesatlas.org/. [2021-11-14].

2. Wang JP, Chen YY, Gong QH, An YL, Shen XX, Li GW. Breakthrough in diabetes and cardiovascular disease prevention: a 30-year study of diabetes prevention in Daqing. Sci Sin Vitae 2018;48(8):902 - 8. http:// dx.doi.org/10.1360/N052018-00045. (In Chinese).

3. GBD. Global health data exchange. 2021. http://ghdx.healthdata.org/ gbd-results-tool\#qt-gbd_results_tool_header-ui-tabs1. [2021-11-14].

4. GBD 2019 Diseases and Injuries Collaborators. Global burden of 369 diseases and injuries in 204 countries and territories, 1990-2019: a 
systematic analysis for the Global Burden of Disease Study 2019. Lancet 2020;396(10258):1204 - 22. http://dx.doi.org/10.1016/S0140-6736 (20)30925-9.

5. Mao AY, Lei HC. Health promotion projects in Singapore - a survey report on Singapore. Chin J Prev Contr Chron Non-commun Dis 2003;11(2):91 - 2. http://dx.doi.org/10.3969/j.issn.1004-6194.2003.02. 031. (In Chinese).

6. Li XM, Xia YJ. Research on chronic disease prevention and control strategies at home and abroad. J Pub Health Prev Med 2021;32(3):11721. https://kns.cnki.net/KCMS/detail/detail.aspx?dbcode=CJFD \&db name $=$ CJFDLAST2021 \&filename $=$ FBYF202103027\&uniplatform $=O V$ ERSEAS_EN\&v=A4ZND6suFF1DcCivvDCYsal0iEuLtS_0YffiSyKLx9 uHR6Jfv0Wj3qS-94UpmEvX. (In Chinese).

7. Levy DT, Benjakul S, Ross H, Ritthiphakdee B. The role of tobacco control policies in reducing smoking and deaths in a middle income nation: results from the Thailand SimSmoke simulation model. Tob Control 2008;17(1):53 - 9. http://dx.doi.org/10.1136/tc.2007.022319.

8. TEE E-Siong. Promoting healthy eating and active living in Malaysia. In: Proceedings of the dietary nutrition physical activity and health 11 th annual symposium of danone institute China. Guangzhou: Danone Nutrition Center of China Center for Disease Control and Prevention. 2008. https://kns.cnki.net/kcms/detail/detail.aspx?dbcode=CPFD\&db name $=C P F D 0914 \&$ filename $=$ DNZX200811001003\&uniplatform $=$ NZ KPT\&v=RFrvcEWwk-lxEdEk6dixP8v3MxP6uKcc3VqUkD2xKby Rdb2J2ewhnTjSg8jIUWPjFG3jDRRLfUo\%3d. (In Chinese).

9. Healthy lifestyle for all. 2021. http://www.jiankang121.cn. [2021-1114]. (In Chinese). 